\title{
IMPROVED NEGATIVE SELECTION BASED DECISION BASED MEDIAN FILTER FOR NOISE REMOVAL
}

\author{
Sarmandip Kaur ${ }^{1}$, Navneet Bawa $^{2}$ \\ M.Tech Scholar,ACET Manawala Amritsar \\ simarsarai89@gmail.com \\ Associate Professor,ACET,Manawala,Asr
}

\begin{abstract}
For de-noising an image, various filtering techniques are used but they have contradiction between noise removal and Edge preservation in an image. This de-noising contradiction has no efficient solution and they have to formulate a tradeoff between the two contradictory aspects. In this paper we purpose a new technique that remove the noise and also preserve edges from image. This paper has proposed a new approach which will use decision tree structure to replace the noisy pixel in given window. The proposed technique seems to be effective as it will replace the noisy pixel with its best suitable alternative which is evaluated using negative selection based algorithm. The comparative analysis has clearly shown that the proposed technique outperforms over the available techniques.
\end{abstract}

\section{KEYWORDS}

Decision based median filter, negative selection algorithm, and salt and pepper noise.

\section{Council for Innovative Research}

Peer Review Research Publishing System

Journal: INTERNATIONAL JOURNAL OF COMPUTERS \& TECHNOLOGY

Vol.14, No.8

www.ijctonline.com, editorijctonline@gmail.com 


\section{INTRODUCTION}

Impulse noise is type of noise that is normally degrades digital images, as well as grayscale digital images. Impulse noise is a set of random pixels which has a very high contrast compared to surroundings. Generally impulse noise appears as a pepper of bright or dark spots on the image, and the usually these spots have quite high contrast towards their surroundings areas. The major factor that reduces the quality of the image is Noise. Noise hides the essential details of images. To improve the image qualities, we have to remove noises from the images with no loss of image information. An image containing salt and pepper noise will have dark pixels (Pepper) in bright regions and bright pixels (Salt) in dark regions. This type of noise can be caused by dead pixels, analog-to-digital converter errors, bit errors in transmission etc.

\section{NOISE AND TYPES OF NOISE}

Noise: - Image noise is random variation of brightness or color information in images. Image noise is an undesirable byproduct of image capture that adds spurious and extraneous information. Images acquired through modern sensors may be contaminated by a variety of noise sources. Noise refers to stochastic variations as opposed to deterministic distortions such as shading or lack of focus. Various types of noises are described as follows:

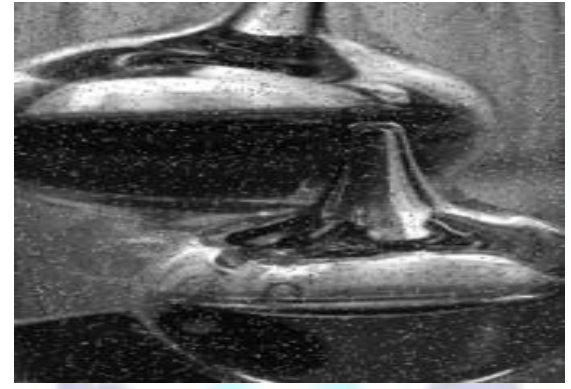

Fig-1

2.1 Salt and Pepper Noise: - Impulse noise is sometimes called salt-and-pepper noise. An image containing salt-and-pepper noise will have dark pixels in bright regions and bright pixels in dark regions. This type of noise can be caused by analog-to-digital converter errors, bit errors in transmission etc.

2.2 Shot Noise:-The dominant noise in the darker parts of an image from an image sensor is usually that caused by statistical quantum fluctuations, that is, variation in the number of photons sensed at a given exposure level. This noise is known as photon short noise.

2.3. Speckle Noise:-Speckle noise is a multiplicative noise. This type of noise occurs in almost all logical imaging systems such as laser, acoustics and SAR (Synthetic Aperture Radar) imagery. The source of this noise is attributed to random interference between the coherent returns. Fully developed speckle noise has the characteristic of multiplicative noise. Speckle noise follows a gamma distribution and is given as

$$
F(g)=\frac{g^{\alpha-1}}{(\alpha-1) ! a^{\alpha}} e^{\frac{-g}{a}}
$$

Where variance is $a^{2} \alpha$ and $g$ is the gray level.

2.4. Amplifier Noise:-The typical model for this type of noise is additive, Gaussian, and independent of the signal. In modern well-designed electronics, amplifier noise is generally negligible. The most common exception to this is in color cameras where more amplification is used in the blue color channel than in the green channel or red channel leading to more noise in the blue channel.

\section{IMAGE DE-NOISING}

Image de-noising is very important task in image processing for the analysis of images. De-noising methods can be linear as well as non-linear. Where linear methods are fast enough, but they do not preserve the details of the images, whereas the non- linear methods preserve the details of the images. Filters play a major role in the image restoration process. Noisy image is individually filtered by various filters such as

\subsection{Switching Median Filter}

Switching median filter is one of the popular median filtering techniques or also known as decision based median filter. Switching median filter checks each input pixel whether it has been corrupted by impulse noise or not. Then it changes only the intensity of noisy pixel, while left the other pixels unchanged. Normally, switching median filter works in two stages. The first stage is for noise detection, while the second stage is for noise reduction. 


\subsection{Hybrid Median Filter}

Hybrid median filter is windowed filter of nonlinear type that easily removes impulse noise while preserving edges detail. In comparison with basic version of the median filter hybrid filter has better corner preserving characteristics. The basic idea behind filter is for any elements of the signal (image) apply median technique several times varying window shape and then take the median of the got median values. The hybrid median filter takes two medians: in an "X" and in a "+" centered on the pixel. The output is the median of these two medians and the original pixel value.

\subsection{Decision Based Filter}

In decision based filter, the pixel value within the window are sorted in ascending order and the middle element of the window is taken as median value of that window. If the processing pixel takes the value of minimum and maximum gray levels, then it is consider as corrupted pixel and it is replaced by median of its neighboring pixels. If the median of the neighboring pixels also takes the value of 0 or 255 , then the processing pixel is replaced by the value of its neighboring pixel value. The drawback of this algorithm is at high noise density the neighboring pixel is used repeatedly for replacing the noisy pixel which degrades the quality of restored images, called streaking effect.

\subsection{Adaptive Median Filter}

The Adaptive Median Filter is used to eliminate the problems faced with the standard median filter. The basic difference between median and in the Adaptive Median Filter is that, in the Adaptive filter the size of the window surrounding each pixel is not fixed. This variation depends on the median of the pixels in the present window. If the median value is a 0 or 255 , then the size of the window is expanded. Otherwise, further processing is done on the part of the image within the current window specification

\subsection{Recursive Median Filter}

The recursive median (RM) filter is a modification of the switched median filter. Recursive Median filtering can extract signal roots better than Switched Median filtering. This filter is useful as alternative to Switched Median filtering in some applications. RM filters are implemented by modifying a Switched Median filtering algorithm. The implementation of Recursive Median filters is computationally and structurally more complex than that of Switched Median filters.

\section{NEGATIVE SELECTION ALGORITHM}

The negative selection algorithm (NSA) is one of models in artificial immune systems. . It is based on the discriminatory mechanism of the natural system. The aim of the negative selection algorithm is to classify a bit or string representations of real-world data, termed antigen, as normal or anomalous. In nature, Antigen is anything which is not part of the body itself. The algorithm processes in two steps: learning and testing. The basic idea of the negative selection algorithm is to generate a number of detectors in the complementary space. Then, apply these detectors to classify new, unseen, data as self or non self.

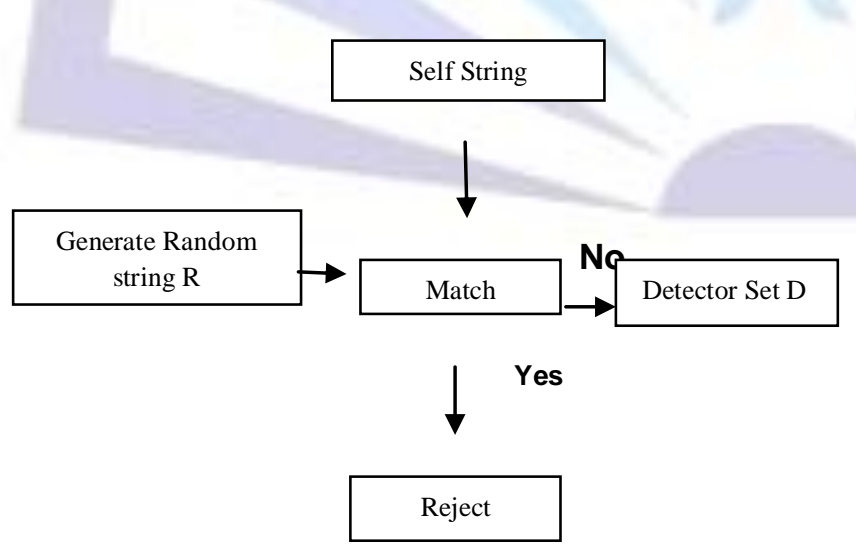

Fig-2 (a) Censoring phase

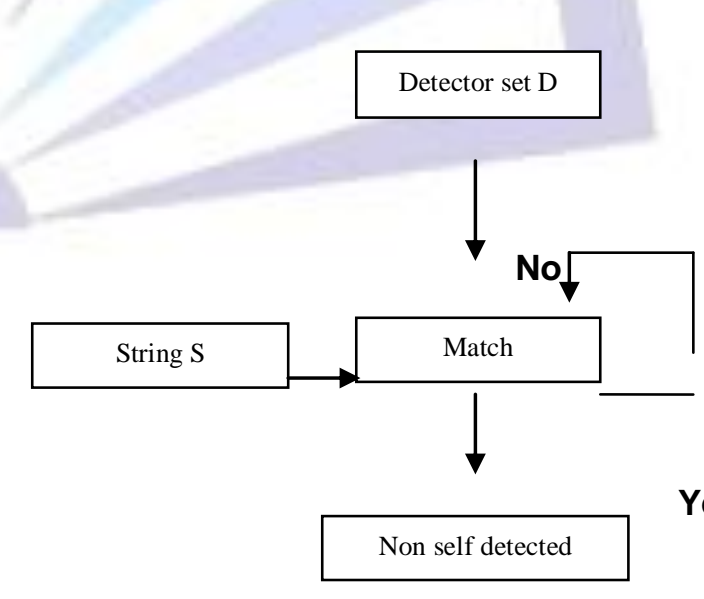

Fig-2 (b) Monitoring Phase 


\section{LITERATURE SURVEY}

Ching-Ta Lu (2014) [1] In this paper, the author aimed at dropping the quantity of musical residual noise by a two-stage speech enhancement approach. In the first phase a preprocessor enhanced noisy speech using an algorithm which combined the two-step-decision-directed and the Virag methods. In the second phase the enhanced speech signal was post-processed by an iterative-directional-median filter to considerably reduce the quantity of residual noise, as maintaining the harmonic spectra.

Nair.S.M. et al.(2013)[2] In this Paper an efficient direction based adaptive weighted switching median filter for restoration of images degraded by high density impulse noise is proposed. The filtering process consists of two phases - detection phase and a filtering phase. The detection phase in the proposed method uses HEIND algorithm put forward by Fei Duan et al. After detecting noisy pixel positions in the image, filtering algorithm was applied to those detected pixels. Noisy pixels were replaced by a weighted median value of uncorrupted pixels in the filtering window or directional filtering window. DAWSM was an improved switching median filter.

Z.Zhe(2012)[3]This paper presented a novel adaptive detail-preserving filter based on the cloud model (CM) to remove impulse noise. It was called the CM filter. Firstly, an uncertainty-based detector identifies the pixels corrupted by impulse noise. Then, a weighted fuzzy mean filter was used to remove the noise. The experimental results showed that, compared with the traditional switching filters, the CM filter gave a great improvement in image de-noising. In addition, the proposed filter identified the noise pixel without needing to sort the pixel gray values, which massively increased the computational efficiency in noise detection.

S.Chandrika et al. (2014) [4] this paper reviewed the existing de-noising algorithms, such as filtering approach; wavelet based approach, and multiracial approach, and performs their comparative study. Different noise models including additive and multiplicative type were used. They included Gaussian noise, salt and pepper noise, speckle noise and Brownian noise.

Vinothini.R.V. et al(2014)[5] This paper proposed a modified decision based unsymmetrical trimmed median filter algorithm for the restoration of gray scale and color images that were highly corrupted by salt-and-pepper noise from digital images. The proposed algorithm replaces the noisy pixel by trimmed median value when other pixel values, 0 's and 255's are present in the selected window and when all the pixel values are 0's and 255 's then the noise pixel is replaced by mean value of all the elements present in the selected window.

Leavline J.E.et al (2013) [6] In this paper, impulse noise removal using the standard median filter and its variants were analyzed. Extensive simulations have been carried out on a set of standard gray scale images and the state of the art median filter variants were compared in terms of the well known image quality evaluation metrics namely mean square error, peak signal to noise ratio and structural similarity index.

Zeng.H.et al. (2012) [7] this paper proposed an algorithm to improve image processing for impulse noise by median filter.The improved algorithm enhanced the detection ability of the single element of an image. Impulse noise was removed by a method of improvement of selection of threshold and filtering templates of median filter.

Jourabloo.A. et al. (2012)[8] In this paper author proposed a new method of noise removal which is applied on images corrupted by impulse noise. This new algorithm has a good trade-off between quantitative and qualitative properties of the recovered image and the computation time. In this new method, the corrupted pixels are replaced by using a median filter or, they are estimated by their neighbors' values.

J. Umamaheswari et al. (2011) [9] proposed a new method based on the hybridization of wavelet and relaxed median filter for de-noising of noisy medical images. The algorithm focuses on to reduce speckle and salt \& pepper noise from scan devices used in medical imaging. The real implementation shows that the new model is more efficient in terms of Peak Signal to Noise Ratio, Mean Square Error and Elapsed Time when compared with existing filters.

Y. Seop Hwang et al. (2013) [10] discussed hybrid median filter in order to remove impulse noises while preserving edges which further helps in improving image quality. Real implementation of the proposed method predicts the effectiveness of the hybrid median filter for rejecting the impulse noises. The performance of the hybrid median filter is evaluated in terms of peak signal to noise ratio and the processing time.

Z. Afrose (2012) [11] discussed performance of different filters such as standard median filter, relaxed median filter, wiener filter ,centre weighted median and averaging filter to remove Salt \& pepper, Gaussian and Speckle noise from compound images. The performance of the different filters with the applied noises using compound images are compared and analyzed according to PSNR value. 
Nain K.A.et al. (2014) [12]This paper compared mixed noise removal techniques such as: Peer Group averaging (PGA), Vector Median Filter (VMF), Vector Direction Filter (VDF), Fuzzy Peer Group Averaging (FPGA), and Fuzzy Vector Median Filter (FVMF) on the basis of performance metrics such as Peak Signal to Noise Ratio (PSNR), Mean Absolute Error (MAE), Mean Square Error (MSE). In this paper a comparative study of mixed de-noising techniques is done.

Lyad F. Jafar et al. (2013) [13] described that the boundary discriminative noise detection (BDND) was one powerful example in this class of Switching median filters. However, there were some issues related to the filtering step in the BDND algorithm that may degrade its performance. In this paper, we proposed two modifications to the filtering step of the BDND algorithm to address these issues. The modifications basically loosen the condition imposed on expanding the filtering window and include the spatial information of the pixels in the filtering process.

Sheena Govindan et al. (2011) [14] has presented a novel method for the removal of random valued impulse noise. The proposed method consisted of two stage detection where the noisy pixels were detected followed by filtering which replaces only the noisy pixels.

\section{PROPOSED METHODOLOGY}

The proposed methodology comprises of the combination of decision based switching median filter and Negative Selection algorithm based on soft computing technique. The proposed method will reduce salt and pepper noise in a corrupted image at high noise densities with greater edge preservation.

Proposed Algorithm:-Following figure shows the flowchart of the proposed algorithm:

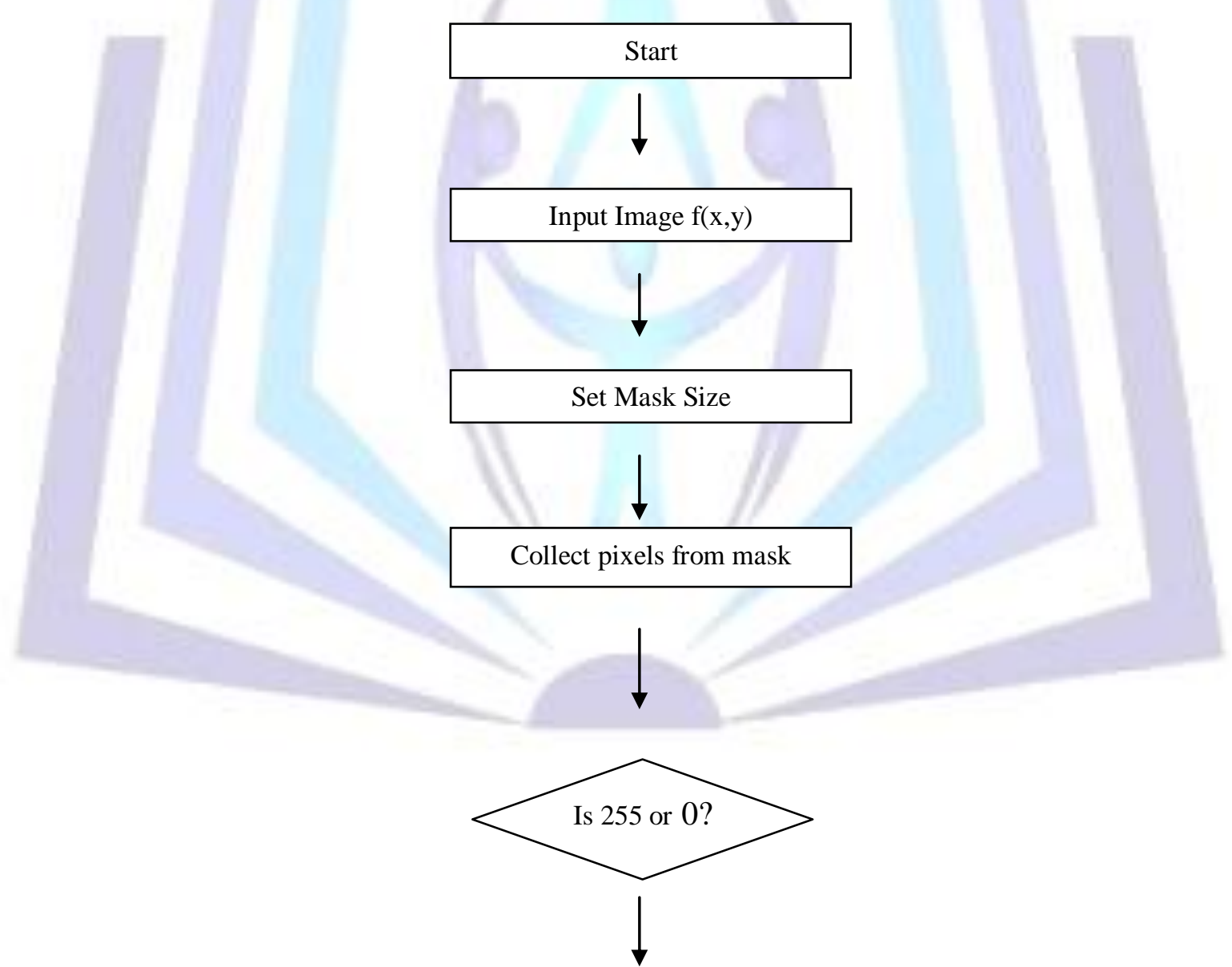

Eliminate pixels 0 or 255

Collect the remaining pixels 


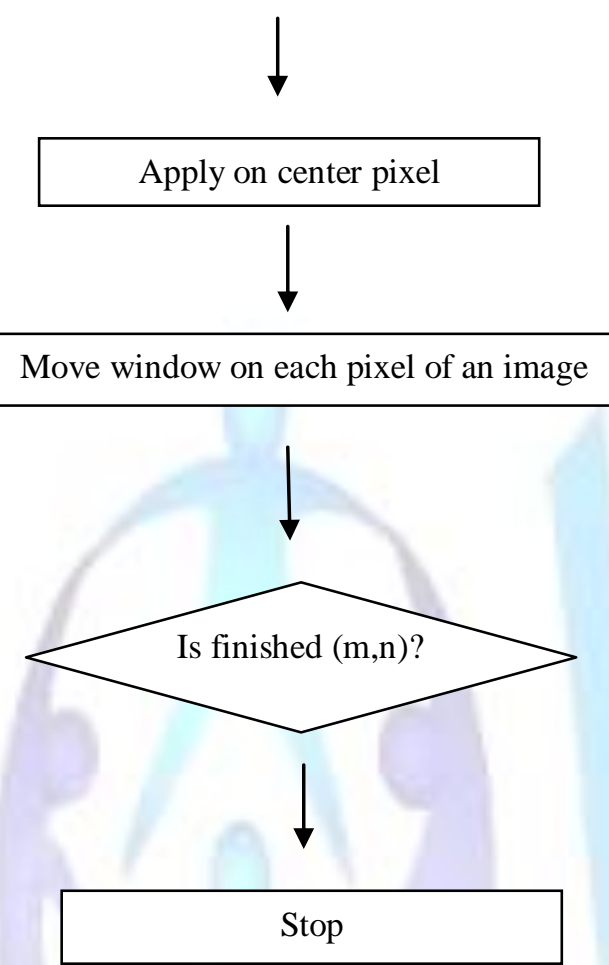

Steps in Proposed Algorithm:

Step 1: Select image from computer memory into current program.

Step 2: Select the dimension size of an image.

Step 3: Repeat the following steps until end of file is not obtained.

Step 4: Collect all the pixels from mask.

Step 5: Check whether pixel values 0 or 255 are present or not.

Step 6: Eliminate all pixel values 0 or 255 and collect the remaining pixels.

Step 7: Evaluate the replacement using soft computing.

Step 8: Obtain the current value of median and apply on center pixel.

Step 9: Move filter on each pixels of an image.

Step 10: When all the corrupted pixels are removed we will obtain the filtered image.

\section{RESULT AND DISCUSSIONS}

\subsection{Experimental Set-Up}

In order to implement the proposed algorithm, design and implementation has done in MATLAB using image processing toolbox. Results of our proposed algorithm are as showed in Fig 3 and 4:- 


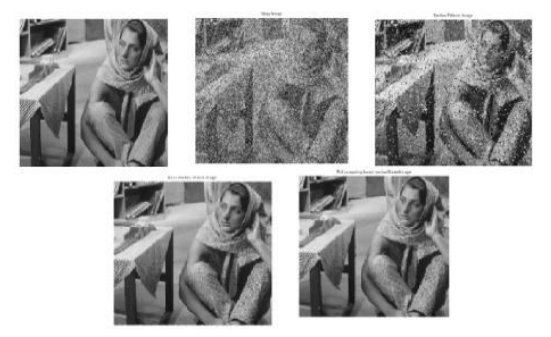

Fig(3) (a)Input image(b).Noisy Image(c)Median filter image (d).Base image(e) Proposed technique image
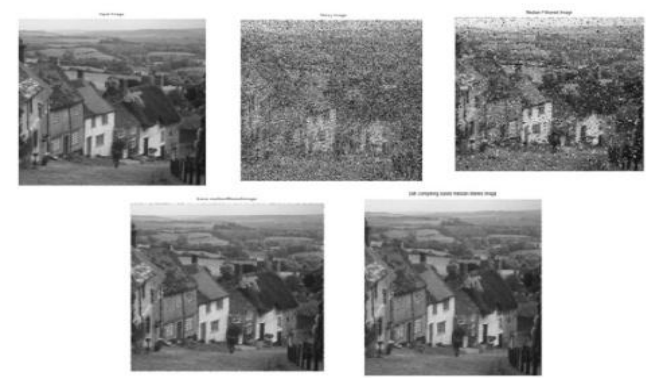

Fig(4) (a)Input image(b).Noisy Image(c)Median filter image (d).Base image(e) Proposed technique image

\subsection{Performance Evaluation}

In this section evaluations of quantitatively performance of existing filtering methods and proposed method are done using various parameters such as Peak Signal to Noise Ratio (PSNR), Mean Squared Error (MSE) and Root Mean Square Error. The images are corrupted by salt and pepper noise with noise density of $70 \%$ has been used. We compare the proposed filter with the noisy image, standard median filter; Decision based median filter and Soft computing algorithm. Both qualitative and quantitative measures will be checked using different tables and graphs for each image by using different evaluation parameters as described above.

\section{Point Signal to Noise Ratio Evaluations (PSNR)}

The goal of proposed method is to increase PSNR as much as possible. Table 1 clearly shows that PSNR is maximum using proposed filter.

Table-1:PSNR

\begin{tabular}{|l|l|l|l|}
\hline $\begin{array}{l}\text { Image } \\
\text { no. }\end{array}$ & $\begin{array}{l}\text { Noisy } \\
\text { image } \\
(70 \%)\end{array}$ & $\begin{array}{l}\text { Median } \\
\text { filter }\end{array}$ & $\begin{array}{l}\text { Edge } \\
\text { Preserving } \\
\text { Healing }\end{array}$ \\
\hline 1. & 6.8969 & 9.8726 & 25.3433 \\
\hline 2. & 6.7977 & 9.6718 & 21.6570 \\
\hline 3. & 6.6810 & 9.6164 & 22.0349 \\
\hline 4. & 6.7665 & 9.7593 & 29.2659 \\
\hline 5. & 6.1998 & 9.2761 & 28.7356 \\
\hline 6. C & 6.7611 & 9.8281 & 26.5774 \\
\hline
\end{tabular}




\section{Mean Squared Error Evaluations (MSE)}

Less is the value of MSE more is the quality of image. Table 2 and Graph 2 clearly indicate that MSE is less using the proposed algorithm. Thus proposed filter provides best results.

Table 2:MSE

\begin{tabular}{|l|l|l|l|}
\hline $\begin{array}{c}\text { Image } \\
\text { no. }\end{array}$ & $\begin{array}{l}\text { Noisy } \\
\text { image } \\
(70 \%)\end{array}$ & $\begin{array}{l}\text { Median } \\
\text { filter }\end{array}$ & $\begin{array}{l}\text { Edge } \\
\text { Preserving } \\
\text { Healing }\end{array}$ \\
\hline 1. & 13286 & 6696 & 192 \\
\hline 2. & 13593 & 7013 & 444 \\
\hline 3. & 13963 & 7103 & 407 \\
\hline 4. & 13691 & 6873 & 77 \\
\hline 5. & 15599 & 7682 & 87 \\
\hline $6 . \quad$ C & 13708 & 6765 & 143 \\
\hline
\end{tabular}

\section{Root Mean Squared Error Evaluations (RMSE)}

Less is the value of RMSE more is the quality of image. Table 3 and Graph 3 clearly indicate that RMSE is less using the proposed algorithm. Thus proposed filter provides best results.

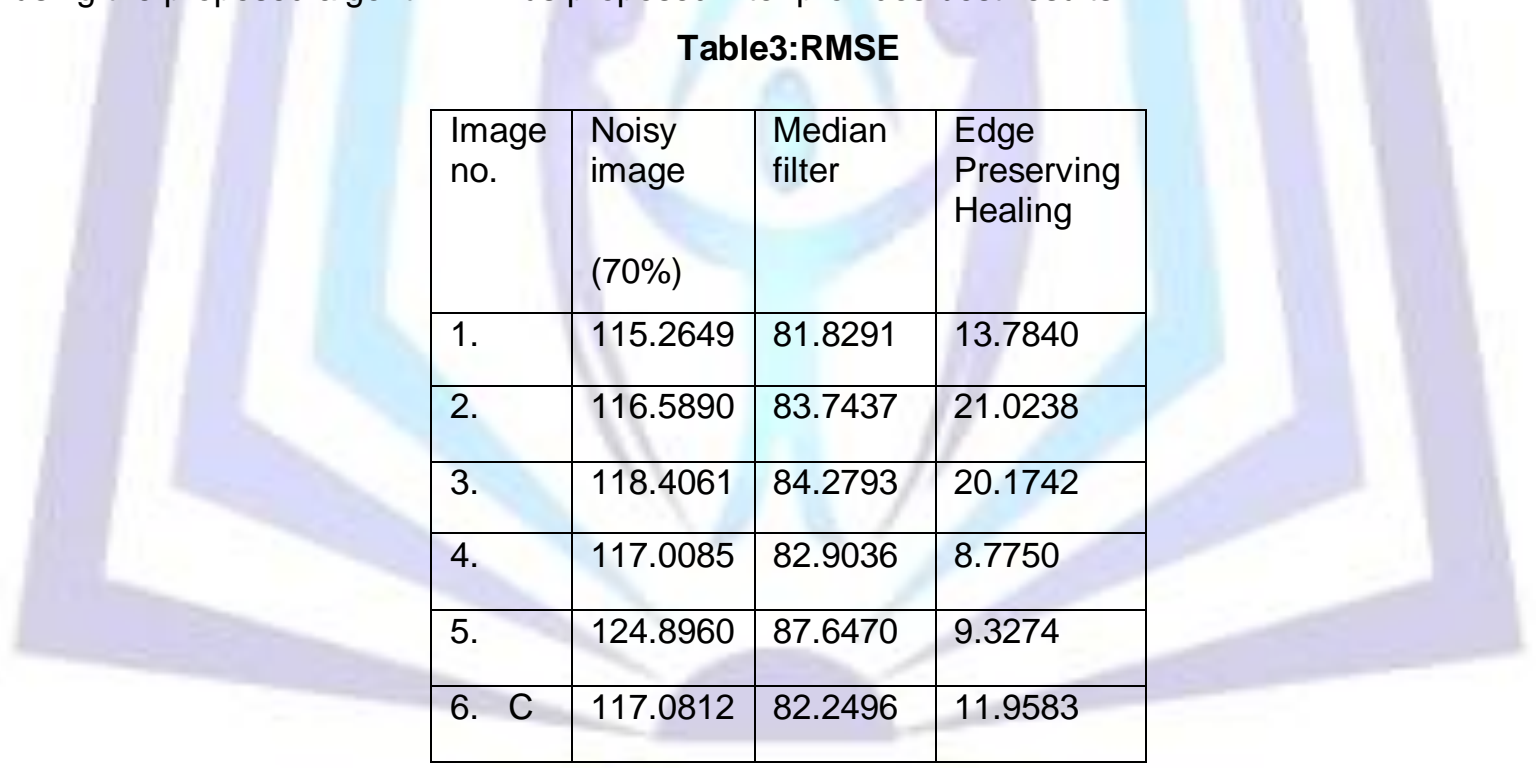

\section{CONCLUSION AND FUTURE WORK}

This paper has proposed a new approach which will use decision tree kind of structure to replace the noisy pixel in given window. The proposed technique seems to be effective as it will replace the noisy pixel with its best suitable alternative. Proposed method is divided into two parts: (1) Algorithm will evaluate the center pixel's value i.e. whether or not it is equal to 0 or 255 if yes then we will go to find the alternative noise free value for the same else window will switched further. (2) This part will find the neighborhood pixels of the center value and see whether all are having 0 or 255 as its value or not. If no then median will be evaluated and replaced with center value and window will switched further else we will use decision tree to evaluate the value by taking the global median and recently evaluated median to replace the same. Global median will be replaced when no median is found recently. The comparative analysis has clearly shown that the proposed technique outperforms over the available techniques. This work has not considered any other kind of noise so in near future we will use different kind of noise to evaluate the effectiveness of the proposed technique. 


\section{References}

1. Lu, Ching-Ta. "Noise reduction using three-step gain factor and iterative directional-median filter." Applied Acoustics 76 (2014): 249-261

2. Nair, Madhu S., and PM Ameera Mol. "Direction based adaptive weighted switching median filter for removing high density impulse noise." Computers \& Electrical Engineering 39.2 (2013): 663-689.

3. Zhou, Zhe. "Cognition and removal of impulse noise with uncertainty." Image Processing, IEEE Transactions on 21.7 (2012): 3157-3167.

4. Saxena, Chandrika, and Deepak Kourav. "Noises and Image Denoising Techniques: A Brief Survey."

5. Vinothini, V. R., and P. Thangaraj. "Modified Decision Based Algorithm Unsymmetric Hybrid Trimmed Median Filter Approach for Removing Salt and Pepper Noise in Ultrasound Images."

6. Leavline, E. Jebamalar, and D. Asir Antony Gnana Singh. "Salt and Pepper Noise Detection and Removal in Gray Scale Images ,An Experimental Analysis." International Journal of Signal Processing, Image Processing and Pattern Recognition 6.5 (2013): 343-352

7. Zeng, Hanglin, et al. "An Improved Algorithm for Impulse Noise by Median Filter." AASRI Procedia 1 (2012): 6873.

8. Jourabloo, A., A. H. Feghahati, and M. Jamzad. "New algorithms for recovering highly corrupted images with impulse noise." Scientia Iranica 19.6 (2012): 1738-1745.

9. J Umamaheswari and Dr.G.Radhamani, "Hybrid Denoising method for removal of mixed noise in medical images", International Journal of Advanced Computer Science and Applications, vol. 3, no. 5, pp. 44-47, 2012.

10. Yo-Seop Hwang, Hyun-Woo Kim, Jang-Myung Lee, "Noise removal of LRF for $3 D$ map building using the superposition median filter”, Advanced Intelligent Mechatronics (AIM), pp. 770-775,2012.

11. Zinat Afrose, "Relaxed Median Filter: A Better Noise Removal Filter for Compound Images", International Journal on Computer Science and Engineering (IJCSE), vol. 4, no.7, pp. 1376-1382, 2012.

12. Nain, Ajay Kumar, et al. "A Comparative Study of Mixed Noise Removal Techniques." International Journal of Signal Processing, Image Processing \& Pattern Recognition 7.1 (2014).

13. Jafar, Iyad F., Rami A. AINa'mneh, and Khalid A. Darabkh. "Efficient Improvements on the BDND Filtering Algorithm for the Removal of High-Density Impulse Noise." Image Processing, IEEE Transactions on 22, no. 3 (2013): 1223-1232.

14. Govindan, Sheena, and S. Saravanakumar. "Removal of Impulse Noise Using Noise Adaptive Fuzzy Switching Median Filter." In IJCA Proceedings on International Conference on VLSI, Communications and Instrumentation (ICVCI)\}, pp. 39-42

15. Guiyang Li, Tao Li, Jie Zeng, Haibo li." An Outlier Robust Negative Selection Algorithm Inspired by Immune Suppression"In JOURNAL OF COMPUTERS, VOL. 5, NO. 9, SEPTEMBER 2010 